\title{
Development of a Synthetic Promoter for Macrophage Gene Therapy
}

\author{
WEIJING HE, ${ }^{1}$ MEI QIANG, ${ }^{2}$ WUQIONG MA,${ }^{1}$ ANTHONY J. VALENTE, ${ }^{1}$ MARLON P. QUINONES, ${ }^{1}$ \\ WEN WANG,${ }^{3}$ ROBERT L. REDDICK, ${ }^{4}$ QIFU XIAO, ${ }^{1}$ SEEMA S. AHUJA,${ }^{1}$ ROBERT A. CLARK, ${ }^{1}$ \\ GREGORY L. FREEMAN, ${ }^{1}$ and SENLIN LI ${ }^{1,2}$
}

\begin{abstract}
Macrophages have the potential to deliver therapeutic genes to many target tissues. Macrophage-specific synthetic promoters (SPs) generated by random ligation of myeloid/macrophage cis elements had activity up to 100-fold that of a native macrophage promoter in macrophage cell lines, but were minimally active in nonmyeloid cells. Mouse bone marrow cells (BMCs) transduced ex vivo with lentivectors expressing green fluorescent protein (GFP) driven either by an SP (SP-GFP) or a cytomegalovirus (CMV) promoter (CMV-GFP) were used for syngeneic transplantation of lethally irradiated mice. Blood leukocytes showed stable GFP expression for up to 15 months after transplantation. SP-GFP expression was selective for $\mathrm{CD}_{11 b^{+}}$macrophages, whereas CMV-GFP expression was observed in erythrocytes, as well as in both $\mathrm{CD}_{11 b^{+}}$and $\mathrm{CD}^{-11 b^{-}}$leukocytes. Furthermore, SP-GFP expression was much stronger than CMV-GFP expression in CD11b ${ }^{+}$macrophages. apo $\mathrm{E}^{-/-}$BMCs transduced with the lentiviral vector encoding human apoE were used to transplant $\mathrm{apoE}^{-/-}$mice. Macrophage expression of apoE from 10 to 26 weeks of age significantly reduced atherosclerotic lesions in recipient apoE $\mathrm{E}^{-1-}$ mice. Thus, the novel SPs, especially when combined with lentivectors, are useful for macrophage-specific delivery of therapeutic genes.
\end{abstract}

\section{OVERVIEW SUMMARY}

Macrophages are differentiated ultimately from hematopoietic stem cells (HSCs). By genetic manipulation of HSCs, macrophages can be directed to express therapeutic genes, using macrophage-specific promoters. To improve macrophage gene therapy we developed a panel of synthetic promoters by random combination of known native myeloid/ macrophage cis elements. These synthetic promoters showed much higher promoter activity than native macrophage promoters in myeloid/macrophage cells, whereas in nonmyeloid cells, their promoter activity was negligible. When packaged into a lentiviral vector, a selected synthetic promoter drove high-level expression of reporter genes selectively in macrophages and this expression persisted until the recipient animals were killed. As a therapeutic proof of principle, we used the synthetic promoter to drive apoE expression in the macrophages of atherosclerosis-prone apoE $\mathrm{E}^{-/-}$mice. This approach was shown to significantly reduce atherosclerotic lesion size. The novel synthetic pro- moter, which has high activity and is selectively expressed in myeloid/macrophage cells, can be used to enhance therapeutic macrophage-specific gene expression.

\section{INTRODUCTION}

$\mathbf{M}$ ACROPHAGES ARE DIFFERENTIATED from hematopoietic stem cells (HSCs) in bone marrow and recruited to most tissues in the body. The accessibility of HSCs and the broad range of locations to which macrophages emigrate, in addition to their high intrinsic biosynthetic capacity, make these cells an attractive vehicle for delivery of therapeutic genes (Clarke and Gordon, 1998). At the same time, macrophages play a crucial role in the generation of immune responses (Gordon, 1992) and in the pathogenesis of chronic inflammatory diseases such as atherosclerosis (Paulos et al., 2004), and are involved in the growth and metastasis of tumors (Coussens and Werb, 2001). For example, overexpression of genes associated with lipid metabolism, such as apolipoprotein AI (apoAI) ( $\mathrm{Ru} \mathrm{Su}$ et al.,

${ }^{1}$ Department of Medicine, ${ }^{2}$ Department of Pharmacology, ${ }^{3}$ Department of Urology, and ${ }^{4}$ Department of Pathology, University of Texas Health Science Center at San Antonio, and South Texas Veterans Health Care System, Audie L. Murphy Division, San Antonio, TX 78229. 
2003), and knockdown of inflammatory genes such as CCR2 (Guo et al., 2003) in macrophages can inhibit atherogenesis.

In macrophage gene therapy, HSCs are mobilized from bone marrow, isolated by apheresis, and transduced ex vivo to establish the stable integration into a chromosome of an expression cassette of the gene(s) of choice. After being autologously reinfused these transduced HSCs (Bank, 2003; Trobridge et al., 2005) form various lineages of blood cells, including macrophages. Because they are under the control of macrophage-specific promoters, the therapeutic genes should be expressed at high levels only in macrophages. Therefore, strong macrophage promoters are valuable in this potential therapeutic process. Widespread expression of a therapeutic gene driven by a ubiquitous promoter may cause unforeseeable adverse effects, even though broadly active promoters may have advantages in certain conditions. Unrestricted activity of a promoter (especially a viral promoter) integrated into a chromosome may also activate neighboring genes and increase the chance of tumorigenesis (Brenner and Malech, 2003).

Several macrophage promoters have been characterized including the sheep visna virus long terminal repeat (Bellosta et al., 1995), the microsialin (CD68) promoter (Li et al., 1998), the chicken lysozyme minigene construct (Whitman et al., 2002), and the scavenger receptor-A (SA) promoter (Horvai et al., 1995). However, these native macrophage promoters may not be strong and/or specific enough for the purpose of gene therapy. In addition, some of them may be too large to be incorporated into a viral vector. Efforts have been made to create synthetic promoters with high levels of activity in skeletal muscle for potential therapeutic advantage (Li et al., 1999). Here we describe the development of a synthetic myeloid-specific promoter that is small in size, has high-level macrophageselective activity, and can be used successfully in lentiviral vectors to transduce HSCs, leading to sustained expression of the transgene in transplanted animals. We also demonstrate that SPdriven apoE constructs are effective in reducing atheroma development in apoE-null mice.

\section{MATERIALS AND METHODS}

\section{Promoter-reporter constructs}

All DNA segments of the promoter were derived according to published data and inserted into pGL3-Basic vector (Promega, Madison, WI). The CD68 promoter contained 342 bp of sequence $5^{\prime}$ to the ATG initiation codon of the CD68 gene, in addition to the CD68 first intron (IVS-1) (Gough and Raines, 2003). The SA promoter consisted of the enhancer $(-4.5$ to $-4.1 \mathrm{~kb}$ upstream of the major transcriptional start site) and core promoter (bp -245 to +46$)$ (Wu et al., 1994; Horvai et al., 1995). The $\mathrm{p} 47^{\text {phox }}$ promoter was from bp -86 to +52 of the $47^{\text {phox }}$ gene (Li et al., 1997). The CD11b promoter covered the gene from bp -1704 to +83 (Dziennis et al., 1995). The macrophage colony-stimulating factor receptor (M-CSFR, or CSF1R) promoter contained $5 \mathrm{~kb}$ upstream and 300 bp downstream of the gene (Hahn et al., 1998). A similar cytomegalovirus (CMV)-luciferase reporter construct was also made. All constructs were diagnosed by restriction enzyme digestion and confirmed by sequencing.

\section{Cell culture and transient transfection}

Human monocytic cell lines THP-1 and Mono Mac-1, and the T lymphocyte Jurkat cell line, were cultured in RPMI 1640 (Invitrogen, Carlsbad, CA) plus 10\% fetal bovine serum. Mouse macrophage cell lines RAW264.7, J774, and WEHI-3, human intestinal epithelial cell line Caco-2, cervix epithelioid carcinoma cell line HeLa, human embryonic kidney cell line 293, and mouse osteoblast cell line Oct-1 were cultured in Dulbecco's modified Eagle's medium (DMEM; Invitrogen) plus $10 \%$ fetal bovine serum. All cells were transfected with FuGENE 6 reagent (Roche Applied Science, Indianapolis, IN) according to the manufacturer's instructions. Luciferase reporter constructs were cotransfected together with a Renilla vector (pRL-null; Promega) for control of transfection efficiency as described previously (Li et al., 2002). Promoter activity is represented as relative luciferase activity (promoter/vector or control construct).

\section{Promoter library creation}

Complementary DNA oligonucleotides corresponding to $\mathrm{cis}$ elements that bind to myeloid/macrophage transcription factors were synthesized and annealed as described previously (Li et al., 1997). Double-stranded oligonucleotides of PU.1A, PU.1B, $\mathrm{C} / \mathrm{EBP} \alpha, \mathrm{AML}-1, \mathrm{Sp} 1$, and AP-1 cis elements, along with an NheI linker, at a ratio of 2:2:2:2:1:1:1 were randomly ligated and the products were separated on agarose gels. DNA fragments $100-500 \mathrm{bp}$ in length were collected and inserted upstream of the minipromoter at the NheI site into the pGL3-p4786 reporter construct that we characterized previously ( $\mathrm{Li}$ et al., 1997) to generate a synthetic promoter library. Plasmid DNA from 220 colonies was individually prepared with a QIAprep Spin Miniprep kit (Qiagen, Valencia, CA) and cotransfected with pRL-null (Promega) Renilla reporter DNA (10:1) into THP-1 cells in a 24-well-plate by FuGENE 6 reagent. Transfection was done according to the manufacturer's instructions, except that double amounts of DNA and the reagent were used. Luciferase activity was measured $48 \mathrm{hr}$ later with the Dual-Luciferase assay system (Promega) and a TD-20/20 luminometer (Turner Designs, Sunnyvale, CA). Thirty-eight of 45 initial clones consistently exhibited at least 5-fold higher luciferase activity than the clone transfected with basal control (pGL3p47-86).

\section{Lentiviral vector construction}

The synthetic promoter elements of clone 146 along with the minipromoter $(\mathrm{SP}+)$ were subcloned into the lentiviral vector Lenti-CMV-GFP (CS-CDF-CG-PRE; Tahara-Hanaoka et al., 2002) to replace the CMV promoter. Briefly, SP+ was amplified by polymerase chain reaction (PCR), using sense primer 5'-CGGAATTCGGTACCGAGCTCTTACGC-3' and antisense primer 5'-CGACCGGTACTGGGTGGCCTCCAGTG-3'. PCR conditions were as follows: 30 cycles of $94^{\circ} \mathrm{C}$ for $30 \mathrm{sec}$, $56^{\circ} \mathrm{C}$ for $30 \mathrm{sec}$, and $72^{\circ} \mathrm{C}$ for $1 \mathrm{~min}$. PCR products and LentiCMV-GFP plasmid DNA were digested with EcoRI and AgeI, and then ligated to form Lenti-SP-GFP constructs. To replace the enhanced green fluorescent protein (EGFP) gene in LentiCMV-GFP or Lenti-SP-GFP with apoE3, human apoE3 was amplified by PCR from expression vector pLHESN (Hasty et 
al., 1999) with sense primer 5'-CGACCGGTCGGAAGATGAAGGTTCTG-3' and antisense primer 5'-GGTGTACACGCATGGCTGCAGGCTTC- $3^{\prime}$. PCR conditions were as follows: 30 cycles of $94^{\circ} \mathrm{C}$ for $20 \mathrm{sec}, 58^{\circ} \mathrm{C}$ for $30 \mathrm{sec}$, and $72^{\circ} \mathrm{C}$ for 1 min. The PCR products and vector Lenti-CMV-GFP or LentiSP-GFP were digested with $A g e \mathrm{I}$ and $B s r$ GI restriction enzymes, and then ligated to form Lenti-CMV-apoE or Lenti-SPapoE. All constructs were diagnosed by restriction enzyme digestion and confirmed by sequencing. Plasmid DNA used for transfection was prepared with an EndoFree Plasmid Maxi kit (Qiagen).

\section{Lentiviral particle production}

$293 \mathrm{~T}$ cells were used to produce lentiviral particles. For a $175-\mathrm{cm}^{2}$ flask, $20 \mu \mathrm{g}$ of lentiviral vector (Lenti-CMV-GFP, Lenti-SP-GFP, Lenti-CMV-apoE, or Lenti-SP-apoE), together with three packaging plasmids (12 $\mu \mathrm{g}$ of gag-pol plasmid pMDLg/p.PRE, $3 \mu \mathrm{g}$ of Rev-expressing plasmid pRSV-Rev, and $5 \mu \mathrm{g}$ of vesicular stomatitis virus glycoprotein G [VSV-G] envelope-expressing plasmid pMD.G), were transfected into 293T cells with FuGENE 6 reagent (Roche Applied Science). Culture media containing lentiviral particles were collected at 36 and $72 \mathrm{hr}$, filtered through $0.45-\mu \mathrm{m}$ pore size filters, and then concentrated 1000 -fold by two rounds of ultracentrifugation at $56,000 \times g$ for $2 \mathrm{hr}$ and at $72,000 \times g$ for $1.5 \mathrm{hr}$. Viral pellets were resuspended in StemPro-34 SFM (Invitrogen) and stored at $-80^{\circ} \mathrm{C}$ for future use. The titers of GFP-expressing lentiviral particles, determined by infection in HeLa cells by the end-point dilution method (Sastry et al., 2002), were consistently between $10^{8}$ and $10^{9} \mathrm{IU} / \mathrm{ml}$. All apoE-expressing lentiviral particle production was monitored by parallel production and titer determination of GFP-expressing lentiviral particles.

\section{Animal procedures}

Female mice on a C57BL/6 background, purchased from Jackson Laboratory (Bar Harbor, ME), were maintained in microisolator cages and fed a rodent chow diet (5010; PMI Nutrition International, St. Louis, MO) containing $4.5 \%$ fat. Recipient mice were housed in autoclaved cages with acidified (pH 3.0) drinking water containing neomycin (100 mg/liter) and polymyxin B (10 mg/liter) (Sigma, St. Louis, MO) from 3 days before to 21 days after transplantation. Blood samples were collected by tail vein puncture or from the left ventricle on sacrifice. Cells separated from heparinized blood were processed for fluorescence-activated cell-sorting (FACS) analysis. Serum separated from nonheparinized blood was stored at $-80^{\circ} \mathrm{C}$ for future use. Serum cholesterol and triglyceride levels were determined with clinical diagnostic reagents (Sigma), adapted for a microplate assay.

\section{Bone marrow cell transplantation}

Five hours before transplantation, recipient mice were lethally irradiated (950 cGy) from a cesium $\gamma$ source (Gammacell 40 B irradiator; MDS Nordion, Ottawa, ON, Canada). Donor mice were injected intravenously with 5-fluorouracil (10 $\mathrm{mg} / 10 \mathrm{~g}$ body weight; Sigma) 4 days before sacrifice. Bone marrow cells were harvested from femurs and tibias of donor mice by flushing with StemPro-34 SFM (Invitrogen) supplemented with $2 \%$ fetal bovine serum, penicillin $(100 \mathrm{IU} / \mathrm{ml})$, streptomycin $(100 \mu \mathrm{g} / \mathrm{ml})$, and heparin (5 units/ml). HSCs were enriched in the lymphocyte layer by gradient centrifugation with Lympholyte-M (Cedarlane Laboratories, Hornby, ON, Canada). After washing, enriched HSCs were cultured overnight in StemPro-34 SFM containing interleukin (IL)-3 (6 ng/ml), IL-6 (10 $\mathrm{ng} / \mathrm{ml})$, murine IL- $1 \alpha(10 \mathrm{ng} / \mathrm{ml})$, and stem cell factor (100 $\mathrm{ng} / \mathrm{ml})$ in Petri dishes at concentration of $1 \times 10^{6} / \mathrm{ml}$. The next day, cells were pelleted and resuspended in an appropriate small volume (0.4-1 ml) of StemPro-34 SFM containing the same growth factors as indicated above. Infection was performed at a multiplicity of infection (MOI) of 15 for $5 \mathrm{hr}$ in dishes coated with RetroNectin (Takara Bio, Shiga, Japan) in the presence of protamine sulfate $(8 \mu \mathrm{g} / \mathrm{ml})$. After infection, $2 \times 10^{6}$ cells in $0.2 \mathrm{ml}$ of Hanks' buffer containing $2 \%$ fetal bovine serum (FBS) were transplanted by tail vein injection into a lethally irradiated recipient mouse.

\section{Flow cytometry}

Peripheral blood was taken from the tails of mice at various time points and diluted 1:50 in phosphate-buffered saline (PBS) for FACS analysis of red blood cells (RBCs). The unique features of forward scatter (FSC) and side scatter (SSC) of RBCs and leukocytes were used to set up the threshold, and the gated RBCs were analyzed for GFP expression. For FACS analysis of GFP expression in leukocytes, RBCs were lysed in red blood cell lysis buffer (Sigma). Cell suspensions were washed twice in PBS with $2 \%$ bovine serum albumin (BSA) and then subjected to FACS. For CD11b experiments, cells were stained with 1:100-diluted primary antibody (biotin anti-mouse CD11b; BD Biosciences Pharmingen, San Diego, CA) for 30 min at $4^{\circ} \mathrm{C}$ followed by 1:100-diluted secondary antibody (streptavidin-allophycocyanin [SAv-APC]; BD Biosciences Pharmingen). Two-color flow cytometry analyses were performed with a FACScan (BD Biosciences Immunocytometry Systems, San Jose, CA).

\section{Western blotting}

Human apoE3 was examined by Western blot analysis. Briefly, $2.5 \mu \mathrm{l}$ of serum was diluted to $10 \mu \mathrm{l}$ with PBS and denatured in $5 \times$ sodium dodecyl sulfate (SDS) loading buffer at $95^{\circ} \mathrm{C}$ for $5 \mathrm{~min}$. Denatured samples were loaded onto $12 \%$ polyacrylamide gels. The primary antibody was 1:2000-diluted polyclonal goat anti-human apoE (Biodesign International, Saco, ME), and the second antibody was horseradish peroxidase (HRP)-conjugated rabbit anti-goat IgG (diluted 1:5000). The signal was developed with an enhanced chemiluminescence (ECL) kit (Pierce Biotechnology, Rockford, IL). Some samples were also quantified with an enzyme-linked immunosorbent assay (ELISA) kit (MBL International, Woburn, MA) according to the manufacturer's instructions. Serum was first diluted 1:50 in dilution buffer and then $100 \mu \mathrm{l}$ was used for ELISA.

\section{Peritoneal macrophage cultures}

Peritoneal cells were collected 3 days after intraperitoneal injection of $1 \mathrm{ml}$ of $3 \%$ thioglycolate per mouse, and seeded into 12 -well plates at $1 \times 10^{6}$ cells per well in DMEM-10\% 
FBS. Three hours later, nonadherent cells were removed. The adherent macrophages were cultured for an additional $48 \mathrm{hr}$, and then medium was collected and stored with $1 \mathrm{~m} M$ phenylmethylsulfonyl fluoride (PMSF) at $-80^{\circ} \mathrm{C}$ for Western analysis.

\section{Aorta lesion area quantification}

Recipient mice were killed at 16 weeks posttransplantation. Animals were perfused via the left ventricle with $15 \mathrm{ml}$ of cold PBS containing $1 \mathrm{~m} M$ EDTA and $30 \mathrm{ml}$ of fixative (PBS containing $4 \%$ paraformaldehyde and 5\% sucrose). The heart along with the aortic root was dissected, fixed overnight, and frozen in O.C.T. medium (Sakura Finetek USA, Torrance, CA). Frozen sections were cut $8 \mu \mathrm{m}$ thick from the region of the proximal aorta, starting from the beginning of the aortic sinus (identified by an experienced pathologist) and continued distally. Every third section was collected and 20 sections from each animal were examined. Sections were stained with oil red O (Sigma) and counterstained with hematoxylin. Quantification of lesion area was performed by digitizing morphometry, using software SPOT 3.5.5 (Diagnostic Instruments, Sterling Heights, MI) and reported as mean square micrometers per section.

\section{Statistical analysis}

All values are expressed as means \pm SEM. To compare experimental groups, analysis of variance (ANOVA) followed by two-tailed Student $t$ test or $\chi^{2}$ analysis was used with $p<0.05$ considered significant.

\section{RESULTS}

\section{Comparison of native myeloid/macrophage promoters}

Myeloid/macrophage promoters reported in the literature (Dziennis et al., 1995; Horvai et al., 1995; Li et al., 1997; Hahn et al., 1998; Gough and Raines, 2003) from human CD68, SA, $\mathrm{p} 47^{\text {phox }}$, CD11b, and CSF1R genes were inserted into the pGL3Basic reporter vector (Promega) and tested and compared for strength (Fig. 1A and B) and specificity (Fig. 1C) in human myeloid/monocytic THP-1 cells versus nonmyeloid HeLa cells in transient transfection assays. To avoid the influence of different transfection efficiencies with each cell line, we used the ratio of promoter activity in myeloid cells to that in nonmyeloid cells as myeloid/macrophage specificity (Fig. 1C). This allowed us to make comparisons among the various promoters. Not surprisingly, the CMV promoter demonstrated much higher activity than these myeloid promoters in HeLa cells, but poor myeloid specificity. Among the myeloid promoters, the CD68 promoter showed the highest activity in THP-1 cells, but the lowest myeloid/macrophage specificity, whereas the p47phox promoter not only drove high expression of the reporter gene, but also conferred the highest myeloid specificity. Of note, these ratios cannot be taken as absolute values because of variance in transfection efficiency in the two cell lines, but provide a useful index of specificity. Because our strategy for making the synthetic promoter aimed to increase promoter activity and to retain or enhance promoter specificity, the $\mathrm{p} 47^{p h o x}$ promoter was chosen as the minipromoter for promoter synthesis and as a comparator for promoter selection. The small size (138 bp) of the p47phox promoter was also an important factor in this choice.

\section{Creation and screening of synthetic promoter library}

To generate macrophage-specific promoters, myeloid-specific cis promoter elements for PU.1, C/EBP $\alpha$, and AML- 1 and myeloid-associated cis elements for $\mathrm{Sp} 1$ and AP-1 (Tenen et al., 1997; Clarke and Gordon, 1998) (Fig. 2A) were randomly ligated and inserted into a luciferase reporter construct upstream of the $47^{\text {hhox }}$ basal promoter (Fig. 2B), which we have characterized previously (Li et al., 1997). There are two different categories of PU.1 binding sites: GAGGAA and GGAGAA
A

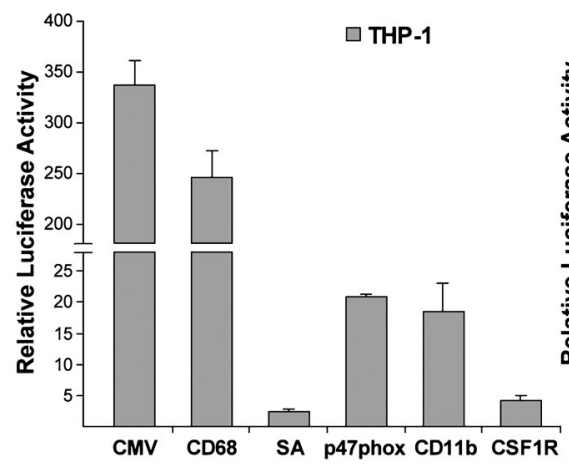

B

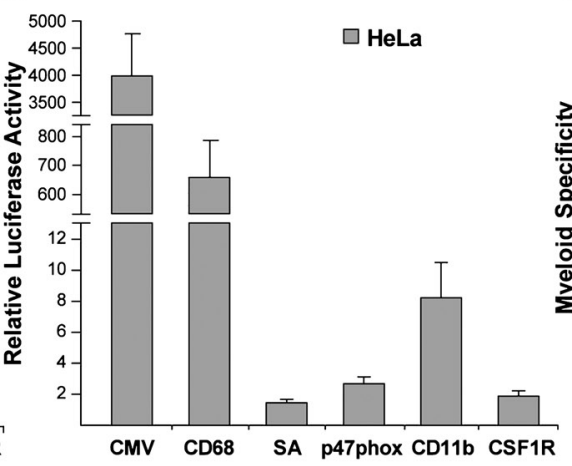

C

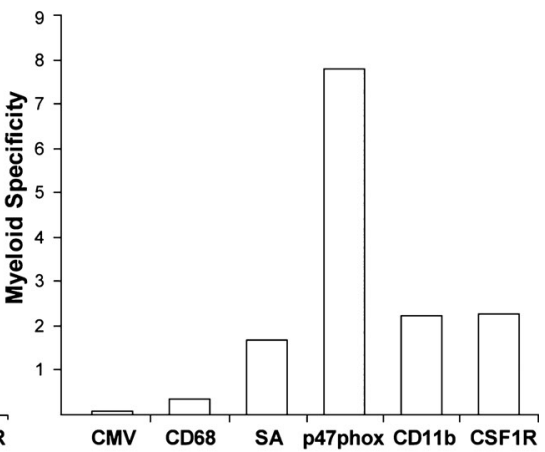

FIG. 1. Comparison of promoter activity: Relative promoter activity in myeloid THP-1 cells (A) and nonmyeloid HeLa cells (B). Cells were cotransfected with the indicated promoter reporter constructs and a Renilla reporter vector (PRL-null; Promega) and luciferase activity was measured $48 \mathrm{hr}$ later by Dual-Luciferase analysis. Luciferase activity was further normalized to that obtained with the pGL3-Basic vector. (C) Myeloid specificity of promoters (THP-1/HeLa). The ratio of promoter activity in myeloid (THP-1) cells to that in nonmyeloid (HeLa) cells was used as an expression of myeloid specificity. 


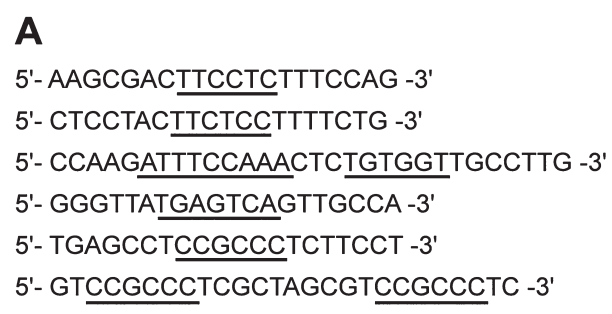

\author{
PU.1A ( $\left.p 47^{\text {phox }}\right)$ \\ PU.1B (CD11b) \\ $\mathrm{C} / \mathrm{EBP} \alpha+\mathrm{AML} 1(\mathrm{CSF}-1 R)$ \\ AP1 ( $\left.p 67^{\text {phox }}\right)$ \\ Sp1 (CD11b) \\ Nhel-linker
}

FIG. 2. Construction of the SPs. (A) Oligonucleotides used to make SPs. Sequences of the cis elements were derived from native myeloid promoters of the genes named in parentheses. The NheI linker was flanked by an $\mathrm{Sp} 1$ site on both sides. Core or consensus motifs are underlined. (B) SP structure. Randomly ligated SP DNA fragments were inserted at the NheI site into pGL3-p47-86 reporter plasmid. (C) Order and orientation of the components. Selected SPs with the highest promoter activity are indicated with symbols.
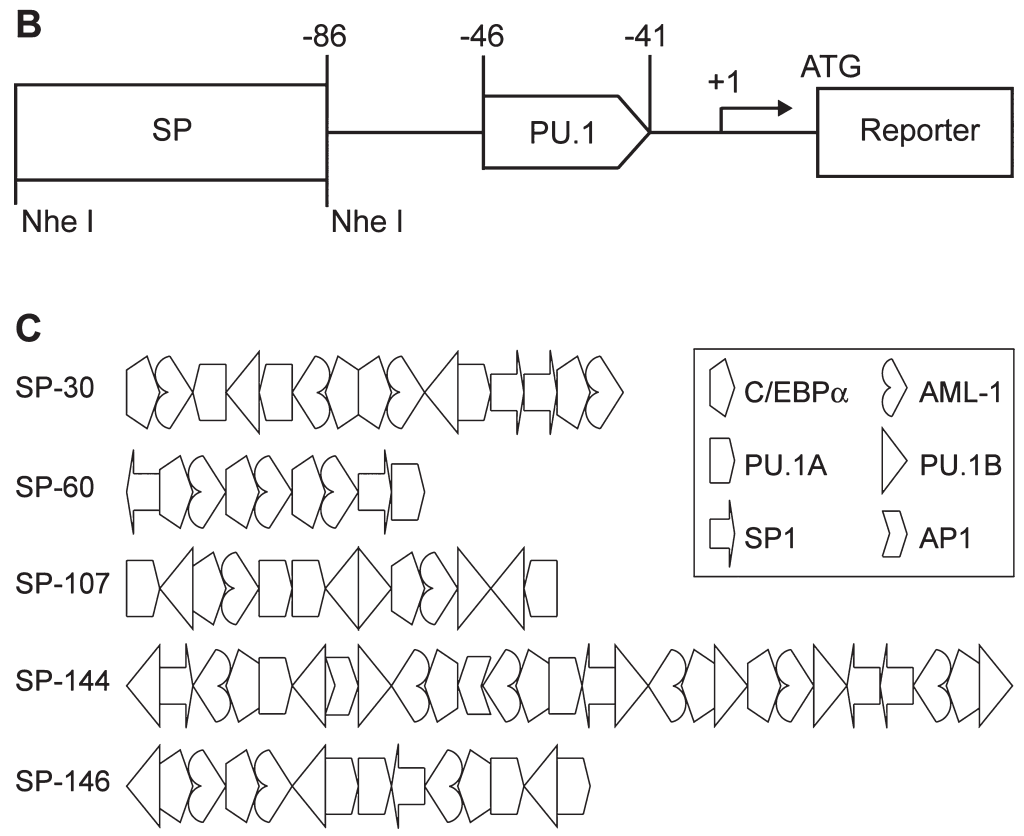

(TTCCTC and TTCTCC on the complementary strands; see Fig. 2A). Both were chosen and designated as PU.1A and PU.1B, respectively. Native sequences adjacent to the core motif of each cis regulatory element were included to avoid loss of potentially important sequences and constructs were restricted to either 20 or 30 bp in length, such that regulatory elements would appear on the same face of the DNA helix when reassembled. This library was screened for promoter activity in human THP-1 monocytic cells by transient transfection and measurement of luciferase activity. Thirty-eight independent clones that consistently exhibited promoter activity at least 5fold higher than the basal control (pGL3-p47-86) were identified. Those with the highest levels of activity were sequenced (Fig. 2C; GenBank accession nos. DQ107381, DQ107382, and DQ107383) and further characterized.

\section{Macrophage specificity of synthetic promoters}

After preliminary screening, the strength and specificity of eight of the synthetic promoters were evaluated by transient transfection of several macrophage and nonmacrophage cell lines. In human monocytic cell lines THP-1 and Mono Mac-1, mouse macrophage cell line RAW264.7 (Fig. 3A-C), and J774 and WEHI-3 (data not shown), luciferase activity of the promoters was extremely high, 2- to 50-fold over that of the p47phox and CD11b promoters, 10 - to 200 -fold above that of the CSF1R
(Ramprasad et al., 1996) and SAR (Horvai et al., 1995) promoters, and higher than or at least comparable to that of the CMV promoter. In contrast, in human intestinal epithelial cell line Caco-2, cervix epithelioid carcinoma cell line HeLa, and human embryonic kidney cell line HEK293 (Fig. 3D) and in T lymphocyte Jurkat cells and mouse Oct-1 osteoblasts (data not shown), specific luciferase activity of the promoters was quite low compared with the CMV promoter. Thus, these synthetic promoters (SPs) were specific for macrophage cell lines and had high activity compared with native promoters. On the basis of these data, we selected SP146 for the remainder of the expression studies.

\section{Synthetic promoter-based lentiviral vectors support long-term gene expression}

To assess long-term promoter activity in vivo, we transplanted bone morrow stem cells transduced ex vivo with synthetic promoter-based lentiviral vectors (SP-GFP). We adapted the method of Pawliuk et al. (2001), in which HSCs transduced with lentiviral vectors retain their stem cell characteristics. At 16 weeks after transplantation GFP expression in peripheral leukocytes (Fig. 4A-C) and red blood cells (Fig. 4D) was analyzed by FACS. As shown in Fig. 4D, few red blood cells $(1.5 \%)$ expressed GFP protein in SP-GFP-transplanted mice, but almost all red blood cells $(95.0 \%)$ expressed GFP in CMV- 
A

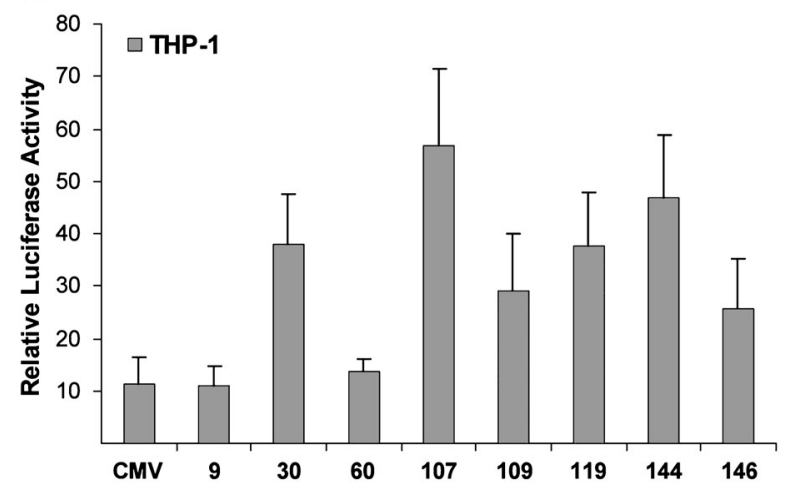

B

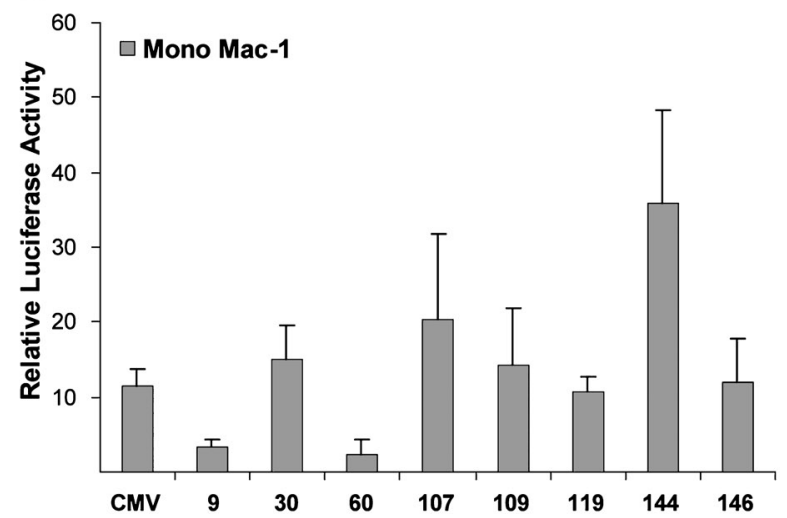

C
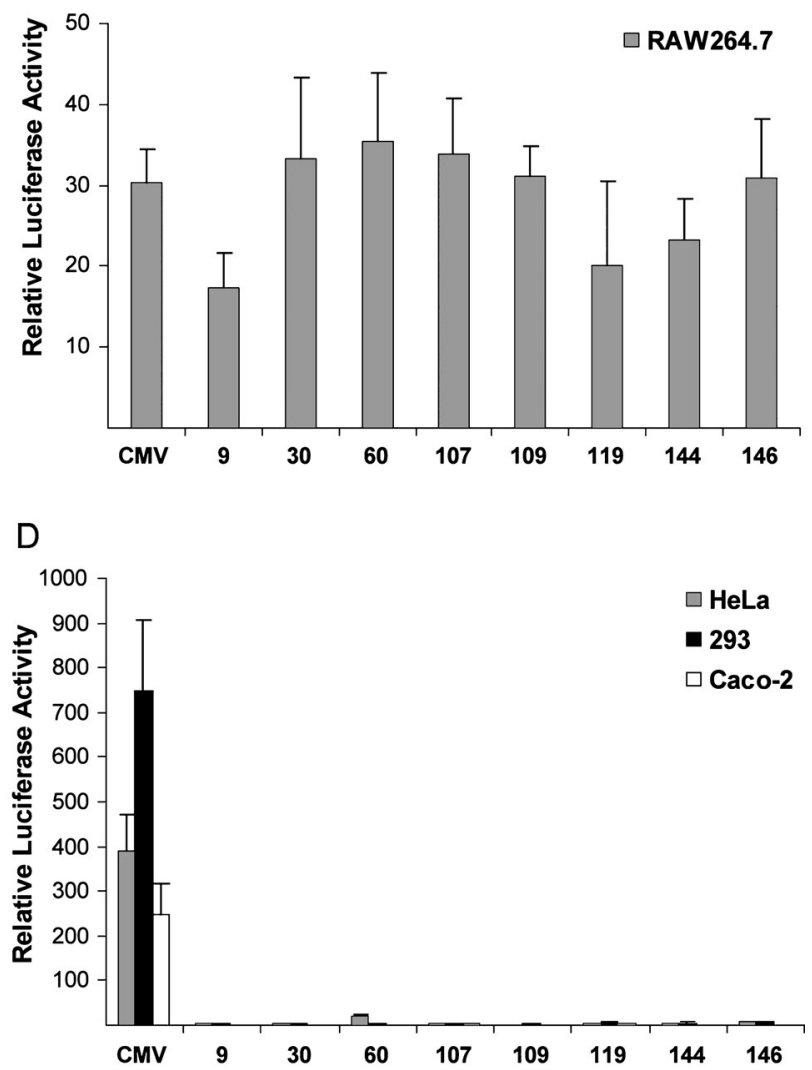

FIG. 3. Macrophage specificity of SP activity tested by transient transfection. Monocyte/macrophage cell lines THP-1 (A), Mono Mac-1 (B), and RAW264.7 (C) and nonmacrophage cell lines HeLa, 293, and Caco-2 (D) were transfected and promoter activity was measured $48 \mathrm{hr}$ later by Dual-Luciferase analysis as described in Fig. 1. Luciferase activity was further normalized to that obtained with the basal vector (pGL3-p47-86). SP reporter constructs are designated by clone number. Of note, larger scales for luciferase activity in (D) indicate the higher transfection efficiency in these cells.

GFP-transplanted animals. Meanwhile, as shown in Fig. 4B and E, SP-GFP was expressed in most monocyte/macrophage cells $(68.9 \%)$, as identified by antibody to Mac-1/CD11b, and was expressed in a much smaller fraction of CD11 ${ }^{-}$cells $(20.4 \%)$. Most of the cells with low mean fluorescence intensity (MFI) in the GFP gate, and that were CD11b negative, had low SSC and intermediate FSC, suggesting they were likely lymphocytes (data not shown). Thus, the promoter was preferentially active in cells of the myeloid/macrophage lineage. The CMV-GFP construct was expressed in $>80 \%$ of both $\mathrm{CD} 11 \mathrm{~b}^{+}$and CD11b ${ }^{-}$leukocytes (Fig. 4C and E). Importantly, SP-GFP was highly expressed $\left(\mathrm{MFI}, \sim 1 \times 10^{3}\right.$ ) in $\mathrm{CD} 11 \mathrm{~b}^{+}$macrophages (upper right quadrant of Fig. 4B) but was expressed at much lower levels $\left(\sim 6 \times 10^{1}\right)$ in $\mathrm{CD}_{11 b^{-}}$leukocytes (lower right quadrant); whereas CMV-GFP was expressed at moderate levels $\left(\sim 1.5-5.0 \times 10^{2}\right)$ in all types of white blood cells (right half of Fig. 4C). Of note, GFP expression in both SP-GFP- and CMV-GFP-transplanted mice was quite stable over time, although the latter showed a decreasing trend (up to 15 months when the animals were killed; Fig. 4F). These results indicate that lentiviral vector-mediated HSC gene transfer driven by a synthetic macrophage promoter has the potential to provide long-lasting high-level expression of therapeutic genes in target cells.

apoE is reconstituted in apoE $E^{-1-}$ mice by ex vivo-transduced bone marrow

To test potential applications of our synthetic promoter we used atherosclerosis-prone apoE $\mathrm{E}^{-1-}$ mice. apoE is a major component of very low-density lipoproteins (VLDLs), a key function of which is to remove excess cholesterol from the blood and carry it to the liver for processing. It is well established that atherosclerosis development is driven by accumulation of activated macrophages in the arterial wall. Replacement of apoE in the myeloid compartment of apoE $\mathrm{E}^{-/-}$mice is sufficient to prevent the development of atherosclerosis, and apoE synthesized locally by resident macrophages retards the formation of atherosclerotic plaques, possibly by mediating cholesterol efflux (Linton et al., 1995; Boisvert and Curtiss, 1999; Sakai et al., 2002). apoE $\mathrm{E}^{-/-}$mice at 10 weeks of age were lethally irradiated and given tail vein injections of bone marrow cells transduced ex vivo with the lentivector carrying human apoE (Fig. 5A). Serum apoE was examined by Western blot (Fig. 

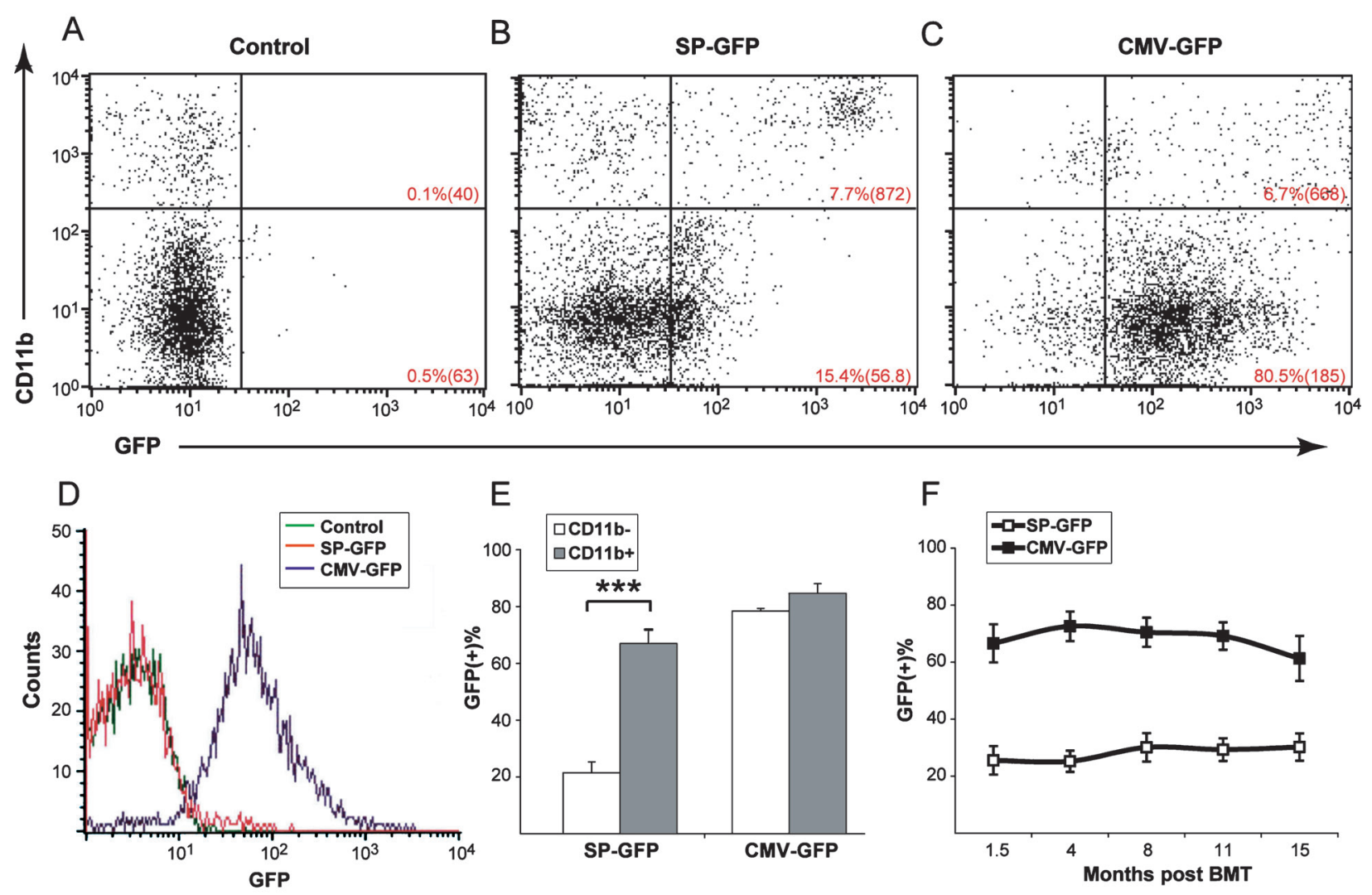

FIG. 4. Lentivector-mediated SPs drive high and stable expression of GFP in macrophages post-BMT. Mice were transplanted with bone marrow cells transduced with either SP-GFP or CMV-GFP lentivectors, and peripheral blood was analyzed by FACS for GFP expression. CD11b was used as a monocyte/macrophage marker. (A-C) GFP expression profiles (dot plot) in leukocytes from mice 4 months post-transplant. Percentages of $\mathrm{GFP}^{+}$leukocytes in each quadrant were indicated and mean fluorescence intensity (MFI) shown in parentheses. (D) GFP expression profiles (histogram) in erythrocytes from the same mice as above. (E) Quantitative comparison of GFP expression in CD11 b ${ }^{+}$and CD11b leukocytes of SP-GFP or CMV-GFP recipients. Data shown are means \pm SEM from seven recipient mice of each group four months post-BMT. ${ }^{*} p<0.001$. (F) GFP ${ }^{+}$cells in peripheral leukocytes of recipient mice at various time points post-BMT. Data shown are means \pm SEM from three or more recipient mice for each group.

5B). As expected, there was no apoE detected in serum from recipient mice of negative controls (Fig. 5B, lane 3). apoE was detected in serum of the recipient mice as early as 3 weeks posttransplantation (data not shown) and remained consistent over time (Fig. 5B) up to 12 months, when the mice were killed (data not shown). Serum apoE in the SP-apoE group was only onefourth to one-third of that in the CMV-apoE group (lanes 7 and 8 versus lanes 4 and 5 in Fig. 5B). This was verified by ELISA, which showed concentrations of serum apoE of $240.6 \pm 99.0$ and $660.8 \pm 214.0 \mathrm{ng} / \mathrm{ml} 16$ weeks posttransplantation in the $\mathrm{SP}-\mathrm{apoE}$ group and the CMV-apoE group, respectively. Notably, the CMV-apoE group had levels of serum apoE similar to those of the positive controls (lanes 4, 5, and 9 in Fig. 5B). Levels of serum apoE among individual mice in each group were comparable (data not shown).

To investigate whether stem cells had been transduced and grafted into the recipients, secondary transplantations were performed. Bone marrow was collected from two mice transplanted with SP-apoE 16 weeks after bone marrow transplantation (BMT) and transplanted into four lethally irradiated 10-weekold apoE $\mathrm{E}^{-/-}$recipient mice. apoE was detected in serum 10 weeks post-BMT in these secondarily transplanted mice (Fig. $5 \mathrm{~B}$, lane 6), and remained measurable for as long as 5 months (data not shown).

\section{Macrophage apoE expression ameliorates atherosclerotic lesions}

Culture medium of peritoneal macrophages from each group was also analyzed by Western blot (Fig. 5C). apoE levels produced by macrophages from SP-apoE and CMV-apoE groups were comparable, although lower than those from positive controls, which had received bone marrow from wild-type mice. However, levels of circulating apoE were about $70 \%$ lower after BMT with the macrophage-specific promoter than with the CMV promoter (Fig. 5B), probably because of the selective activity of the former and the ubiquitous activity of the latter, as evidenced in Figs. 2 through 4.

The effect of gene therapy with SP-apoE lentivirus on atherosclerotic lesion development was examined by quantitative morphometry of sections taken from the root of the aorta 16 weeks after transplantation (Fig. 5D and E). As shown in Fig. 
A

\begin{tabular}{|c|c|c|c|}
\hline Group & Donor mice & $\begin{array}{l}\text { Lentiviral vector } \\
\text { used to infect HSCs }\end{array}$ & $\begin{array}{c}\text { Recipient } \\
\text { mice }\end{array}$ \\
\hline$a$ & ApoE-/- & CMV-apoE & apoE-/- \\
\hline$b$ & ApoE-/- & SP-apoE & apoE-/- \\
\hline c & $\mathrm{ApoE}^{-/-}$ & SP-GFP & apoE-/- \\
\hline$d$ & C57BL/6 & None (Pos C) & apoE $E^{-/-}$ \\
\hline
\end{tabular}

B

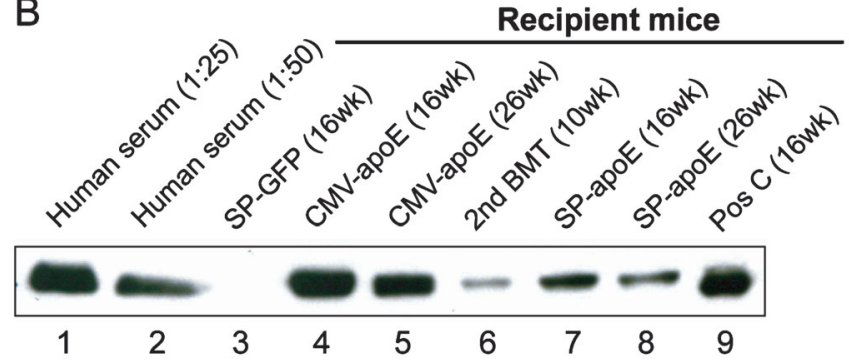

D
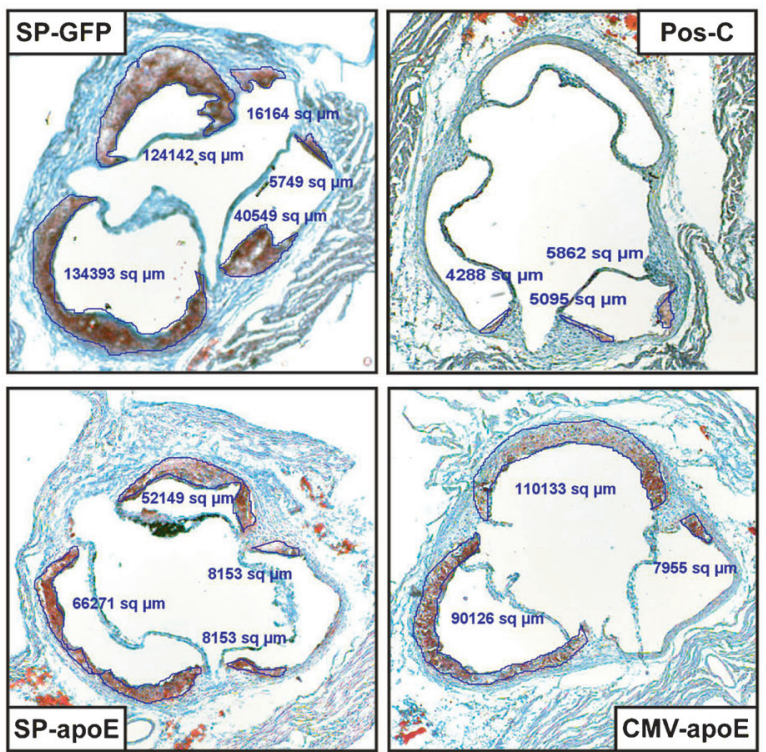

$\mathrm{E}$

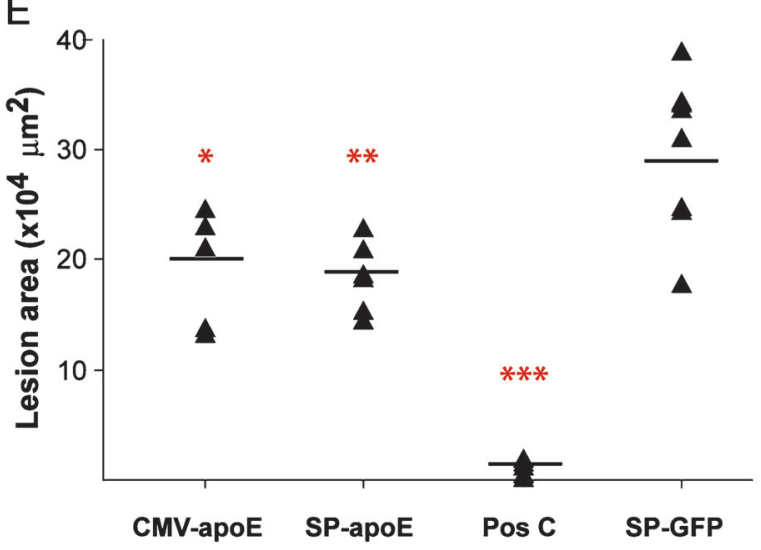

FIG. 5. apoE reconstitution and amelioration of atherosclerosis in apoE $\mathrm{E}^{-/-}$mice. (A) Group design for apoE replacement by BMT. Groups c and d served as negative and positive control (Pos C), respectively. (B) Western analysis of transgene apoE in serum. Lanes 1 and 2, normal human serum diluted as indicated; lanes 3-5 and 7-9, serum from recipients of groups at various time points as indicated; lane 6, a second BMT recipient mouse, which was transplanted with bone marrow of a primary SPapoE recipient. (C) Immunoblot analysis of apoE secreted from cultured peritoneal macrophages. Peritoneal macrophages were collected from recipient mice 16 weeks posttransplantation. Lane 1, human serum diluted as indicated; lanes 2-7, supernatant from different groups as indicated. All supernatants were 20 times concentrated before loading, except that in lane 7 , which was further diluted 1:50. (D) Representative aortic lesion of apoE $\mathrm{E}^{-/-}$mice treated as indicated. Recipient mice were killed 16 weeks post-BMT. Sections $(8 \mu \mathrm{m})$ were cut, starting from the aortic sinus. Every third section was collected and stained with oil red $\mathrm{O}$. The numbers labeled in the image indicate lesion area values from measurement program. (E) Quantitative data were from 20 sections from each animal. The bar in the middle of each distribution plot represents the mean value of the lesion area for that group. $* p<0.05, * * p<0.01$, and $* * * p<0.001$ compared with the control group (SP-GFP).

5E, mice receiving SP-apoE showed a $38.3 \%$ reduction in lesion area compared with control mice receiving SP-GFP $\left(184,533 \pm 32,242 \mu \mathrm{m}^{2}, n=6\right.$ versus $298,853 \pm 69,094 \mu \mathrm{m}^{2}$, $n=8 ; p<0.01)$. Mice receiving CMV-apoE showed a $36.0 \%$ reduction in lesion area $\left(191,434 \pm 52,550 \mu \mathrm{m}^{2}, n=5\right.$ versus $\left.298,853 \pm 69,094 \mu \mathrm{m}^{2}, n=8 ; p<0.05\right)$. There was no significant difference in lesion area between mice transplanted with apoE $\mathrm{E}^{-/-}$bone marrow and control apoE $\mathrm{E}^{-1-}$ mice, indicating a lack of impact of the transplantation procedure itself on atheroma burden (data not shown). Of note, mice transplanted with control apoE ${ }^{+/+}$marrow showed a $96.0 \%$ decrease $(p<0.001)$ in lesion area compared with control mice $\left(10,567 \pm 7078 \mu \mathrm{m}^{2}, n=5\right.$ versus $298,853 \pm 69,094 \mu \mathrm{m}^{2}$, $n=8$ ), consistent with previous reports (Linton et al., 1995). These data imply an inverse correlation between atherosclerotic lesion area and the amount of macrophage-produced local apoE in the lesions. However, serum cholesterol and triglycerides measured pretransplantation, and 7 or 16 weeks posttransplan- 
tation, showed no significant difference among the experimental groups, with the exception of an approximately $50 \%$ reduction of serum cholesterol in mice receiving apoE ${ }^{+/+}$marrow compared with other groups (data not shown).

\section{DISCUSSION}

Using randomly assembled combinations of promoter elements known to be highly active in myeloid cells, we developed a novel promoter that drives high levels of reporter gene expression in human and mouse myeloid/macrophage cell lines. When packaged in a lentiviral vector and transduced into bone marrow stem cells the promoter drove expression of GFP that was restricted to myeloid cells and highest in macrophages. Expression of apoE in macrophages driven by this promoter ameliorated the development of atherosclerosis in apoE-null mice.

We first compared several available macrophage promoters in transient transfection assays. We noted that the SA promoter activity was less active in THP-1 cells than reported by Wu et al. (1994). This might be due to different transfection methods (FuGENE 6 versus DEAE-dextran). However, our study agreed with a published result that the CD68 promoter was approximately 100-fold more active than the SA promoter in these cells (Ramprasad et al., 1996; Li et al., 1998). The $\mathrm{p} 47^{\text {phox }}$ promoter showed much higher macrophage specificity than the CD68 promoter, which has been reported to be useful in a retroviral vector to direct macrophage-specific gene expression in vivo (Gough and Raines, 2003). Moderate macrophage specificity was observed for the CD11b promoter, which has been demonstrated to direct myeloid/macrophage-specific expression of transgene in transgenic mice (Dziennis et al., 1995). Of note, however, another study using a transgenic model showed different results in that the $\mathrm{CD} 11 \mathrm{~b}$ promoter drove transgene expression in both lymphocytes and granulocytes when small DNA sequences were used (Back et al., 1995). The mechanisms underlying these different results will require further elucidation.

Although cells have evolved many strategies to orchestrate gene activation and repression including chromosomal structures and epigenetic features, gene expression in any given cell type and developmental stage is ultimately regulated by cis elements and trans-acting factors. Tissue macrophages are recruited from monocytes in blood. Together with granulocytes these cells are termed myeloid cells. Macrophages and granulocytes share common myeloid-specific cis elements (Clarke and Gordon, 1998). In macrophages transcription of a gene (either endogenous or transgene) can be determined by the presence and combination of myeloid-specific (such as PU.1) and myeloid-associated (such as AP1) cis elements in its promoter region. $\mathrm{Li}$ and colleagues used random ligation of cis elements known to participate in transcriptional regulation in skeletal muscle and screened a series of synthetic promoters strongly active in that tissue (Li et al., 1999). We used a parallel strategy to create a synthetic promoter-reporter library and screened the library in human monocytic cell line THP-1 to select for macrophage specificity. We chose SP146 in this study for further assessment because in myeloid cell lines it exhibited activities comparable to or exceeding the ubiquitously expressed CMV promoter, but had negligible expression in other cell lines.
To facilitate in vivo testing of the synthetic promoter and to mimic potential clinical applications we used a lentiviral vector to transduce HSCs, which were then transplanted into irradiated mice. We chose lentivirus as the vector for HSC macrophage-mediated gene therapy because it is superior for transduction of bone marrow stem cells (Woods et al., 2002; Kondo et al., 2003), is less subject to silencing over the long term in vivo than a murine oncoretroviral vector (Brenner and Malech, 2003; Persons and Nienhuis, 2003; Ellis, 2005), and may not be tumorigenic (Wu et al., 2003; Jacoby, 2005). As can be seen in Fig. 4F, after lentivector delivery, GFP expression driven by the synthetic promoter was stable for up to 15 months, indicating the absence of gene silencing over time, whereas GFP expression driven by the CMV promoter tended to decrease as reported by others (Persons and Nienhuis, 2003). Synthetic promoter expression was seen predominantly in $\mathrm{CD}_{1} 1 \mathrm{~b}^{+}$leukocytes, mainly in macrophages, and was not found in red blood cells. Furthermore, the intensity of GFP expression driven by the synthetic promoter was more than 10 -fold higher than that driven by the CMV promoter. Thus, the combination of lentiviral vector and the synthetic promoter led to robust, durable, and macrophage-specific expression of the reporter gene.

We also considered the possibility that the SP would be active in dendritic cells of macrophage lineage. We transduced HSCs with SP-GFP lentivectors and induced their differentiation into dendritic cells under culture conditions that we have previously described (Quinones et al., 2000). In these cultures, GFP expression was localized primarily to cells expressing the dendritic cell marker CD11c. Cells expressing the lymphoid lineage marker B220, which constituted only a small percentage of the culture, were GFP negative (our unpublished observations). Further studies will help us to determine whether gene expression driven by the SP described in this paper could also be used to target dendritic cells in vivo.

We applied our hematopoietic stem cell-lentivector-synthetic promoter system for delivery of apoE, a physiologically relevant gene, in macrophages. apoE knockout mice have severe hyperlipidemia and develop advanced atherosclerotic lesions. Macrophage-specific replacement of apoE is sufficient to blunt the development of atherosclerotic plaque in apoE $\mathrm{E}^{-/-}$ mice even in the context of a hypercholesterolemic atherogenic lipoprotein profile (Bellosta et al., 1995; Fazio et al., 2002; Gough and Raines, 2003), and an inverse relationship has been found between macrophage production of apoE and atherosclerotic burden, suggesting the importance of local apoE production/concentration in the antiatherogenic effect. However, limiting apoE production to macrophages is not an essential requirement for achieving these benefits and apoE produced by other blood cells may make some contribution. Hasty et al. showed that when the human apoE transgene was expressed in macrophages of apoE $\mathrm{E}^{-1-}$ mice from 5 to 13 weeks of age there was a significant reduction in lesion area, but no effects were detected in mice that expressed apoE from 10 to 26 weeks (Hasty et al., 1999). However, Gough and Raines demonstrated a dramatic decrease (74\%) in atherosclerotic lesions when transgene apoE was expressed in macrophages of apo $\mathrm{E}^{-/-}$mice from 8 to 20 weeks of age (Gough and Raines, 2003). We assessed lesion area in mice that expressed human apoE from 10 to 26 weeks of age with either the synthetic promoter or the 
CMV promoter and found that both sets of animals had reduced lesion area (38.3 or $36.0 \%$; Fig. 5D and E). The results confirm that macrophage-specific delivery of apoE is sufficient to reduce lesion area, and that the synthetic promoter is effective in bringing this about. Macrophage expression of apoE after transplantation of transduced stem cells into apoE-null recipients was comparable in the two groups (Fig. 5C), whereas circulating apoE was much higher in animals transplanted with marrow transduced with the broadly active CMV promoter, compared with the synthetic promoter (Fig. 5B). The different levels of macrophage apoE and circulating apoE present after transduction with the two promoters are consistent with their known activity: SP is active mainly in macrophages, whereas CMV is active in all blood cells.

In addition to the specific role of macrophages in atherosclerosis, they are also an attractive option to deliver other therapeutic genes. Interestingly, we have shown that macrophages can accumulate in substantia nigra, the specific location of injury in the 1-methyl-4-phenyl-1,2,3,6-tetrahydropyridine (MPTP) mouse model of Parkinson's disease. Glial cell-derived neurotrophic factor (GDNF), a dopamine neuron-protective factor, may be delivered to the damaged site by HSC-derived macrophages to ameliorate the disease (our unpublished data). In such a case, macrophage-specific expression of therapeutic genes may be highly desired.

In summary, by randomly combining several myeloid-specific promoter sequences, we have developed a novel synthetic promoter that is highly active in macrophages and that when packaged in a lentiviral vector can be used to drive expression of a physiologically relevant gene, apoE. Expression was sustained over a long time period, and was sufficiently robust to blunt the development of atherosclerosis in apoE-null mice. Further studies will be needed to define more completely the utility of this promoter as a means of exploring mechanisms of human diseases and as a possible therapeutic tool.

\section{ACKNOWLEDGMENTS}

The authors thank Dr. Hiroyuki Miyoshi (RIKEN Tsukuba Institute, Japan) for providing lentivectors CS-CDF-CG-PRE and the three packaging vectors, Drs. Alyssa Hasty and Sergio Fazio (Vanderbilt University) for providing pLHESN vector, Dr. Peter Gough (Harborview Medical Center, Seattle, WA) for CD68S-HA-EGFP (containing the CD68 promoter), Dr. Christopher K. Glass (University of California San Diego) for vector EP-H (containing the SA promoter), Dr. Erik Bonten (St. Jude Children's Research Hospital) for the CSF1R promoter, Dr. Daniel G. Tenen (Beth Israel Deaconess Medical Center/Harvard Medical School) for the CD11b promoter, Dr. Robert Pawliuk (Harvard Medical School) for technical advice, Barry Grubbs for technical assistance with intravenous injection, and Jennifer Sharron as well as Zhenhua Lu for graphic assistance. This work was supported by a research grant from San Antonio Area Foundation, a Grant-in-Aid from the Texas Affiliate of the American Heart Association, the Janey Briscoe Center of Excellence in Cardiovascular Research, the Research Division of the Department of Veterans Affairs, and grant AI20866 from the National Institutes of Health.

\section{REFERENCES}

BACK, A., EAST, K., and HICKSTEIN, D. (1995). Leukocyte integrin $\mathrm{CD} 11 \mathrm{~b}$ promoter directs expression in lymphocytes and granulocytes in transgenic mice. Blood 85, 1017-1024.

BANK, A. (2003). Hematopoietic stem cell gene therapy: Selecting only the best. J. Clin. Invest. 112, 1478-1480.

BELlOSTA, S., MAHLEY, R.W., SANAN, D.A., MURATA, J., NEWLAND, D.L., TAYLOR, J.M., and PITAS, R.E. (1995). Macrophage-specific expression of human apolipoprotein E reduces atherosclerosis in hypercholesterolemic apolipoprotein E-null mice. J Clin. Invest. 96, 2170-2179.

BOISVERT, W.A., and CURTISS, L.K. (1999). Elimination of macrophage-specific apolipoprotein $\mathrm{E}$ reduces diet-induced atherosclerosis in C57BL/6J male mice. J. Lipid Res. 40, 806-813.

BRENNER, S., and MALECH, H.L. (2003). Current developments in the design of onco-retrovirus and lentivirus vector systems for hematopoietic cell gene therapy. Biochim. Biophys. Acta 1640, 1-24.

CLARKE, S., and GORDON, S. (1998). Myeloid-specific gene expression. J. Leukoc. Biol. 63, 153-168.

COUSSENS, L.M., and WERB, Z. (2001). Inflammatory cells and cancer: Think different! J. Exp. Med. 193, F23-F26.

DZIENNIS, S., VAN ETTEN, R.A., PAHL, H.L., MORRIS, D.L., ROTHSTEIN, T.L., BLOSCH, C.M., PERLMUTTER, R.M., and TENEN, D.G. (1995). The CD11b promoter directs high-level expression of reporter genes in macrophages in transgenic mice. Blood 85, 319-329.

ELLIS, J. (2005). Silencing and variegation of gammaretrovirus and lentivirus vectors. Hum. Gene Ther. 16, 1241-1246.

FAZIO, S., BABAEV, V.R., BURLEIGH, M.E., MAJOR, A.S., HASTY, A.H., and LINTON, M.F. (2002). Physiological expression of macrophage apoE in the artery wall reduces atherosclerosis in severely hyperlipidemic mice. J. Lipid Res. 43, 1602-1609.

GORDON, S. The mononuclear phagocyte system. (1992). In: McGee, J.O., ed. Oxford Textbook of Pathology. (Oxford University Press, Oxford) pp. 236-258.

GOUGH, P.J., and RAINES, E.W. (2003). Gene therapy of apolipoprotein E-deficient mice using a novel macrophage-specific retroviral vector. Blood 101, 485-491.

GUO, J., VAN ECK, M., TWISK, J., MAEDA, N., BENSON, G.M., GROOT, P.H.E., and VAN BERKEL, T.J.C. (2003). Transplantation of monocyte CC-chemokine receptor 2-deficient bone marrow into ApoE3-Leiden mice inhibits atherogenesis. Arterioscler. Thromb. Vasc. Biol. 23, 447-453.

HAHN, C.N., DEL PILAR, M.M., ZHOU, X.Y., MANN, L.W., and D'AZZO, A. (1998). Correction of murine galactosialidosis by bone marrow-derived macrophages overexpressing human protective protein/cathepsin A under control of the colony-stimulating factor-1 receptor promoter. Proc. Natl. Acad. Sci. U.S.A. 95, 14880-14885.

HASTY, A.H., LINTON, M.F., BRANDT, S.J., BABAEV, V.R., GLEAVES, L.A., and FAZIO, S. (1999). Retroviral gene therapy in ApoE-deficient mice: ApoE expression in the artery wall reduces early foam cell lesion formation. Circulation 99, 2571-2576.

HORVAI, A., PALINSKI, W., WU, H., MOULTON, K.S., KALLA, K., and GLASS, C.K. (1995). Scavenger receptor A gene regulatory elements target gene expression to macrophages and to foam cells of atherosclerotic lesions. Proc. Natl. Acad. Sci. U.S.A. 92, 5391-5395.

JACOBY, J. (2005). Interview with Dr Verma. Gene Ther. 12, 950-953. KONDO, M., WAGERS, A.J., MANZ, M.G., PROHASKA, S.S., SCHERER, D.C., BEILHACK, G.F., SHIZURU, J.A., and WEISSMAN, I.L. (2003). Biology of hematopoietic stem cells and progenitors: Implications for clinical application. Annu. Rev. Immunol. 21, 759-806.

LI, A.C., GUIDEZ, F.R., COLLIER, J.G., and GLASS, C.K. (1998). The macrosialin promoter directs high levels of transcriptional ac- 
tivity in macrophages dependent on combinatorial interactions between PU.1 and c-Jun. J. Biol. Chem. 273, 5389-5399.

LI, S.L., VALENTE, A.J., ZHAO, S.J., and CLARK, R.A. (1997). PU.1 is essential for p47phox promoter activity in myeloid cells. J. Biol. Chem. 272, 17802-17809.

LI, S.L., VALENTE, A.J., QIANG, M., SCHLEGEL, W., GAMEZ, M., and CLARK, R.A. (2002). Multiple PU.1 sites cooperate in the regulation of $\mathrm{p} 40^{\text {phox }}$ transcription during granulocytic differentiation of myeloid cells. Blood 99, 4578-4587.

LI, X., EASTMAN, E.M., SCHWARTZ, R.J., and DRAGHIA-AKLI, R. (1999). Synthetic muscle promoters: Activities exceeding naturally occurring regulatory sequences. Nat. Biotechnol. 17, 241-245.

LINTON, M.F., ATKINSON, J.B., and FAZIO, S. (1995). Prevention of atherosclerosis in apolipoprotein E-deficient mice by bone marrow transplantation. Science 267, 1034-1037.

PAULOS, C.M., TURK, M.J., BREUR, G.J., and LOW, P.S. (2004). Folate receptor-mediated targeting of therapeutic and imaging agents to activated macrophages in rheumatoid arthritis. Adv. Drug Deliv. Rev. 56, 1205-1217.

PAWLIUK, R., WESTERMAN, K.A., FABRY, M.E., PAYEN, E., TIGHE, R., BOUHASSIRA, E.E., ACHARYA, S.A., ELLIS, J., LONDON, I.M., EAVES, C.J., HUMPHRIES, R.K., BEUZARD, Y., NAGEL, R.L., and LEBOULCH, P. (2001). Correction of sickle cell disease in transgenic mouse models by gene therapy. Science 294, 2368-2371.

PERSONS, D.A., and NIENHUIS, A.W. (2003). Gene therapy for the hemoglobin disorders. Curr. Hematol. Rep. 2, 348-355.

QUINONES, M., AHUJA, S.K., MELBY, P.C., PATE, L., REDDICK, R.L., and AHUJA, S.S. (2000). Preformed membrane-associated stores of interleukin (IL)-12 are a previously unrecognized source of bioactive IL-12 that is mobilized within min of contact with an intracellular parasite. J. Exp. Med. 192, 507-516.

RAMPRASAD, M.P., TERPSTRA, V., KONDRATENKO, N., QUEHENBERGER, O., and STEINBERG, D. (1996). Cell surface expression of mouse macrosialin and human CD68 and their role as macrophage receptors for oxidized low density lipoprotein. Proc. Natl. Acad. Sci. U.S.A. 93, 14833-14838.

RU SU, Y., ISHIGURO, H., MAJOR, A.S., DOVE, D.E., ZHANG, W., HASTY, A.H., BABAEV, V.R., LINTON, M.F., and FAZIO, S. (2003). Macrophage apolipoprotein A-I expression protects against atherosclerosis in ApoE-deficient mice and up-regulates $\mathrm{ABC}$ transporters. Mol. Ther. 8, 576-583.

SAKAI, Y., KIM, D.K., IWASA, S., LIANG, J., WATANABE, T., ONODERA, M., and NAKAUCHI, H. (2002). Bone marrow chimerism prevents atherosclerosis in arterial walls of mice deficient in apolipoprotein E. Atherosclerosis 161, 27-34.

SASTRY, L., JOHNSON, T., HOBSON, M.J., SMUCKER, B., and CORNETTA, K. (2002). Titering lentiviral vectors: Comparison of
DNA, RNA and marker expression methods. Gene Ther. 9, 1155-1162.

TAHARA-HANAOKA, S., SUDO, K., EMA, H., MIYOSHI, H., and NAKAUCHI, H. (2002). Lentiviral vector-mediated transduction of murine CD34- hematopoietic stem cells. Exp. Hematol. 30, 11-17.

TENEN, D.G., HROMAS, R., LICHT, J.D., and ZHANG, D.E. (1997). Transcription factors, normal myeloid development, and leukemia. Blood 90, 489-519.

TROBRIDGE, G., BEARD, B.C., and KIEM, H.P. (2005). Hematopoietic stem cell transduction and amplification in large animal models. Hum. Gene Ther. 16, 1355-1366.

WHITMAN, S.C., RATERI, D.L., SZILVASSY, S.J., CORNICELLI, J.A., and DAUGHERTY, A. (2002). Macrophage-specific expression of class A scavenger receptors in LDL receptor ${ }^{-l-}$ mice decreases atherosclerosis and changes spleen morphology. J. Lipid Res. 43, 1201-1208.

WOODS, N.B., OOKA, A., and KARLSSON, S. (2002). Development of gene therapy for hematopoietic stem cells using lentiviral vectors. Leukemia 16, 563-569.

WU, H., MOULTON, K., HORVAI, A., PARIK, S., and GLASS, C.K. (1994). Combinatorial interactions between AP-1 and ets domain proteins contribute to the developmental regulation of the macrophage scavenger receptor gene. Mol. Cell. Biol. 14, 2129-2139.

WU, X., LI, Y., CRISE, B., and BURGESS, S.M. (2003). Transcription start regions in the human genome are favored targets for MLV integration. Science 300, 1749-1751.

Address reprint requests to: Dr. Senlin $\mathrm{Li}$ University of Texas Health Science Center at San Antonio 7703 Floyd Curl Drive San Antonio, TX 78229-3900

E-mail: lis1@uthscsa.edu

Dr. Gregory L Freeman University of Texas Health Science Center at San Antonio 7703 Floyd Curl Drive San Antonio, TX 78229-3900

E-mail: freeman@uthscsa.edu

Received for publication February 19, 2006; accepted after revision July 26, 2006.

Published online: August 28, 2006. 